\title{
Are Elias 2-27's Spiral Arms Driven by Self-gravity, or by a Companion? A Comparative Spiral Morphology Study
}

\author{
Duncan H. Forgan $^{1,2}$ (D), John D. Ilee ${ }^{3}$ (D), and Farzana Meru ${ }^{3,4,5}$ (D) \\ ${ }^{1}$ SUPA, School of Physics and Astronomy, University of St Andrews, North Haugh, St Andrews KY16 9SS, UK; dhf3@st-andrews.ac.uk \\ ${ }^{2}$ Centre for Exoplanet Science, University of St Andrew, St Andrews KY16 9SS, UK \\ ${ }^{3}$ Institute of Astronomy, University of Cambridge, Cambridge CB3 0HA, USA \\ ${ }^{4}$ Department of Physics, University of Warwick, Gibbet Hill Road, Coventry CV4 7AL, UK \\ ${ }^{5}$ Centre for Exoplanets and Habitability, University of Warwick, Gibbet Hill Road, Coventry CV4 7AL, UK \\ Received 2018 March 29; revised 2018 May 15; accepted 2018 May 18; published 2018 June 7
}

\begin{abstract}
The spiral waves detected in the protostellar disk surrounding Elias 2-27 have been suggested as evidence of the disk being gravitationally unstable. However, previous work has shown that a massive, stable disk undergoing an encounter with a massive companion are also consistent with the observations. We compare the spiral morphology of smoothed particle hydrodynamic simulations modeling both cases. The gravitationally unstable disk produces symmetric, tightly wound spiral arms with constant pitch angle, as predicted by the literature. The companion disk's arms are asymmetric, with pitch angles that increase with radius. However, these arms are not well-fitted by standard analytic expressions, due to the high disk mass and relatively low companion mass. We note that differences (or indeed similarities) in morphology between pairs of spirals is a crucial discriminant between scenarios for Elias 2-27, and hence future studies must fit spiral arms individually. If Elias 2-27 continues to show symmetric tightly wound spiral arms in future observations, then we posit that it is the first observed example of a gravitationally unstable protostellar disk.
\end{abstract}

Key words: hydrodynamics - planet-disk interactions - protoplanetary disks - stars: individual (Elias 227) stars: pre-main sequence

\section{Introduction}

Spiral structures generated by gravitationally unstable protostellar disks play a crucial role in the evolution of protostars and the planetary systems that they eventually host. At the instant of a system's formation, the star mass and disk mass are comparable. This guarantees that the Toomre Parameter (Toomre 1964):

$$
Q=\frac{c_{s} \kappa_{\mathrm{epi}}}{\pi G \Sigma} \sim 1
$$

where $c_{s}$ is the sound speed of the disk gas, $\kappa_{\text {epi }}$ is the epicyclic frequency (which is equal to the angular frequency $\Omega$ if the disk is Keplerian), and $\Sigma$ is the surface density. As such, disks that satisfy $Q \sim 1$ will be unstable to non-axisymmetric perturbations, which undergo swing amplification into spiral structures.

At early times, these structures are typically strong global modes, which can achieve rapid accretion of the disk onto the star via non-local angular momentum transport (Laughlin \& Bodenheimer 1994; Lodato \& Rice 2005; Forgan et al. 2011). Under the appropriate conditions, spiral arms can fragment into gravitationally bound objects, representing a formation channel for low-mass stars, brown dwarfs, gas giant planets, and, in some very rare cases, terrestrial planets (Gammie 2001; Rice et al. 2005; Stamatellos et al. 2007; Forgan \& Rice 2013a; Galvagni \& Mayer 2014; Forgan et al. 2018a).

Constraining both disk fragmentation as a formation mechanism, and protostellar accretion in general, requires us to observe bona fide gravitationally unstable protostellar disks in the wild. For observational campaigns, there are (broadly) two approaches to determining whether or not a protostellar disk is gravitationally unstable-measuring its physical properties and determining $Q$, or studying its morphology for signs of spiral structure.

Determining the physical properties of self-gravitating protostellar disks is a challenging endeavor, as the selfgravitating phase is brief due to rapid disk accretion. As a result, self-gravitating disks remain heavily embedded inside their birth molecular cloud. Further to this, their centrally condensed surface density profiles can frustrate efforts to measure disk masses through measuring continuum dust emission, due to large optical depths even at sub-millimeter wavelengths (Greaves \& Rice 2010; Forgan \& Rice 2013b; Forgan et al. 2016b; Evans et al. 2017).

The detection and characterization of spiral structure, then, may yield a bias-free determination of whether or not a protostellar disk is gravitationally unstable. Spirals in protostellar disks have recently come within the reach of observations. Scattered light measurements have yielded several protostellar disks exhibiting spirals (e.g., Fukagawa et al. 2006; Muto et al. 2012; Benisty et al. 2015; Dong et al. 2016), but these observations only trace structure generated in the disk's upper layers. Gravitationally unstable disks will drive structure in the bulk of the disk material, at all altitudes, down to the midplane.

The arrival of the Atacama Large Millimeter Array (ALMA) has allowed exquisite spatially resolved observations of protostellar disks at sufficiently long wavelengths to probe spiral structure at the disk midplane. A most striking recent example is Elias 2-27-a relatively young low-mass star $\left(M_{*} \sim 0.5-0.6 M_{\odot}\right.$, age $\sim 1$ Myr; Luhman \& Rieke 1999; Natta et al. 2006) hosting a Class II circumstellar disk potentially exhibiting a large mass $\left(M_{\mathrm{disc}} \sim 0.04-0.14 M_{\odot}\right.$, Andrews et al. 2009; Isella et al. 2009; Ricci et al. 2010).

Pérez et al. (2016) presented ALMA observations that showed two large-scale symmetric spiral arms. In their study, 
both arms were simultaneously fitted to pure logarithmic spirals, with identical pitch angle $7^{\circ} .9 \pm 0.4$. Given both its relatively large disk-to-star mass ratio $(q \sim 0.06-0.3)$ and the presence of spiral structure driven at the midplane, it has been suggested that Elias 2-27 is a gravitationally unstable disk system (Meru et al. 2017; Tomida et al. 2017).

Of course, gravitational instability (GI) is not the only mechanism that generates spiral structures. Interactions between a disk and a companion generate tidally driven arms, that (to the eye) can be very similar to arms driven in an isolated GI disk. Meru et al. (2017) addressed this issue by running a suite of smoothed particle hydrodynamics (SPH) simulations of both isolated GI disks, and GI disks that are perturbed by a companion. They show that both isolated and perturbed disks produce spiral structures that, when observed synthetically with ALMA, using the same unsharp masking technique as Pérez et al. (2016), produce images consistent with Elias 2-27's features.

Hall et al. (2018) also considered a range of synthetically observed isolated GI disk simulations, and show that if Elias $2-27$ is an isolated GI disk, then its properties are tightly constrained, where slight changes to its physical properties result either in dissipation of the spirals or fragmentation. This suggests that (a priori) Elias 2-27 is less likely to be an isolated GI disk.

We therefore argue that comparative morphology studies are a crucial orthogonal tool to determine whether GI or a companion is driving spiral structure in a given protostellar disk. In this Letter, we perform such a study on the simulations of Meru et al. (2017) to identify crucial differences in spiral morphologies between isolated and perturbed GI disks. Our results offer several discriminants for determining the nature and origin of spiral structure in massive protostellar disks.

\section{Methodology}

\subsection{Hydrodynamics}

Our hydrodynamic simulations are fully described in Meru et al. (2017), but for completeness we briefly reiterate some salient aspects. The simulations are performed using the 3D SPH code (SPHNG) including heating due to work done and the radiative transfer of energy in the flux-limited diffusion limit (Whitehouse et al. 2005; Whitehouse \& Bate 2006). A detailed description of the code can be found in Meru (2015) and Meru et al. (2017).

Our reference model consists of a $0.5 M_{\odot}$ star, modeled as a sink particle, surrounded by a disk with an initial temperature $(T)$ and surface density $(\Sigma)$ that varies with radius $(R)$ as

$$
T(R)=13.4 \mathrm{~K}\left(\frac{R}{200 \mathrm{au}}\right)^{-0.75},
$$

and

$$
\Sigma(R)=\Sigma_{0}\left(\frac{R}{200 \mathrm{au}}\right)^{-0.75}
$$

respectively, between $R_{\text {in }}=10$ au and $R_{\text {out }}=350$ au. The first simulation is a gravitationally unstable disk (GI), with $\Sigma_{0}=$ $6 \times 10^{-7} M_{\odot} \mathrm{au}^{-2}$, giving a total disk mass of $0.24 M_{\odot}$, and an initial Toomre parameter $Q<1$ beyond $\sim 250 \mathrm{au}$.

In the second simulation (Companion), $\Sigma_{0}=1.96 \times$ $10^{-7} M_{\odot} \mathrm{au}^{-2}$, giving a total disk mass of $0.078 M_{\odot}$, and $Q>2$ at all radii.
We note that for the GI case the disk does evolve, such that $\Sigma \propto R^{-0.5}$. For the Companion simulation we model an $8 M_{\text {Jup }}$ companion initially located at 500 au that is allowed to freely interact with the disk, migrate, and grow. At the time when the simulation is analyzed the companion is $\approx 10 M_{\text {Jup }}$ and located at $\approx 425$ au from the central star. The companion does not drive a gap, which is consistent with the gap opening criteria defined by both Lin \& Papaloizou (1986) and Crida et al. (2006). We note that the presence or absence of a gap makes little difference to our results. Each disk is modeled using 250,000 SPH gas particles, and we assume that the gas and dust are well mixed (see Meru et al. 2017 for further details).

\subsection{Spiral Detection and Morphology}

We use the TACHE code, which utilizes tensor-classification of the simulations to determine which SPH particles reside in spiral structures (Forgan et al. 2016a, 2018b). Briefly, we compute the velocity shear tensor of each particle:

$$
\sigma_{i j}=-\frac{1}{2}\left(\frac{\partial v_{i}}{\partial x_{j}}+\frac{\partial v_{j}}{\partial x_{i}}\right) .
$$

And then compute the tensor's eigenvalues. The number of positive eigenvalues $E$ encodes information about the dimensionality of the flow. For example, particles with $E=1$ indicate their motion is planar, consistent with motion in the undisturbed disk. Particles with $E=2$ indicate 2D filamentary motions (in this case, spiral structure). If the spiral structure is strong enough, particles near the center of the arm will possess $E=3$ (3D collapse).

We can therefore identify particles belonging to the spiral using their $E$ value, and discard the other particles. This allows us to trace the spine of the spiral structure (i.e., the location of maximum density), using a friends-of-friends-like algorithm, which yields a set of $(x, y)$ points for each individual spiral. Each arm is then fitted separately via $\chi^{2}$ minimization to a variety of spiral models (assuming a constant uncertainty of $\sigma=0.1 \mathrm{au}$ for all points). We use Nelder-Mead (amoeba) optimization to obtain said minimum $\chi^{2}$, implemented via scipy.optimize.minimize.

Logarithmic spirals are commonly found in isolated GI disks and in disks driven by encounters with a companion:

$$
r=a e^{b \theta}
$$

where $r$ is cylindrical radius, and $\theta$ is the azimuthal angle. The $a$ parameter determines the initial distance of the spiral from the origin, and $b$ determines the winding properties of the arm. The pitch angle of a logarithmic spiral

$$
\phi=\left|r \frac{d \theta}{d r}\right|^{-1}=\arctan b .
$$

Pure logarithmic spirals (where $b=b_{0}$ is a constant) are typically found in simulations of isolated GI disks, with a constant $\phi \sim 10^{\circ}-15^{\circ}$ (for $q<0.5$ Cossins et al. 2009; Hall et al. 2016; Forgan et al. 2018b). We will label model fits of this type as PURELOG.

We also consider models where the pitch angle varies with radius, as is expected if the spiral is being driven by a companion (Goodman \& Rafikov 2001; Rafikov 2002; Muto et al. 2012). For low-mass companions $\left(M \lesssim 1 M_{\text {Jup }}\right.$ in lowmass disks $\left(M_{d} / M_{*} \lesssim 0.1\right)$, logarithmic spirals are typically 

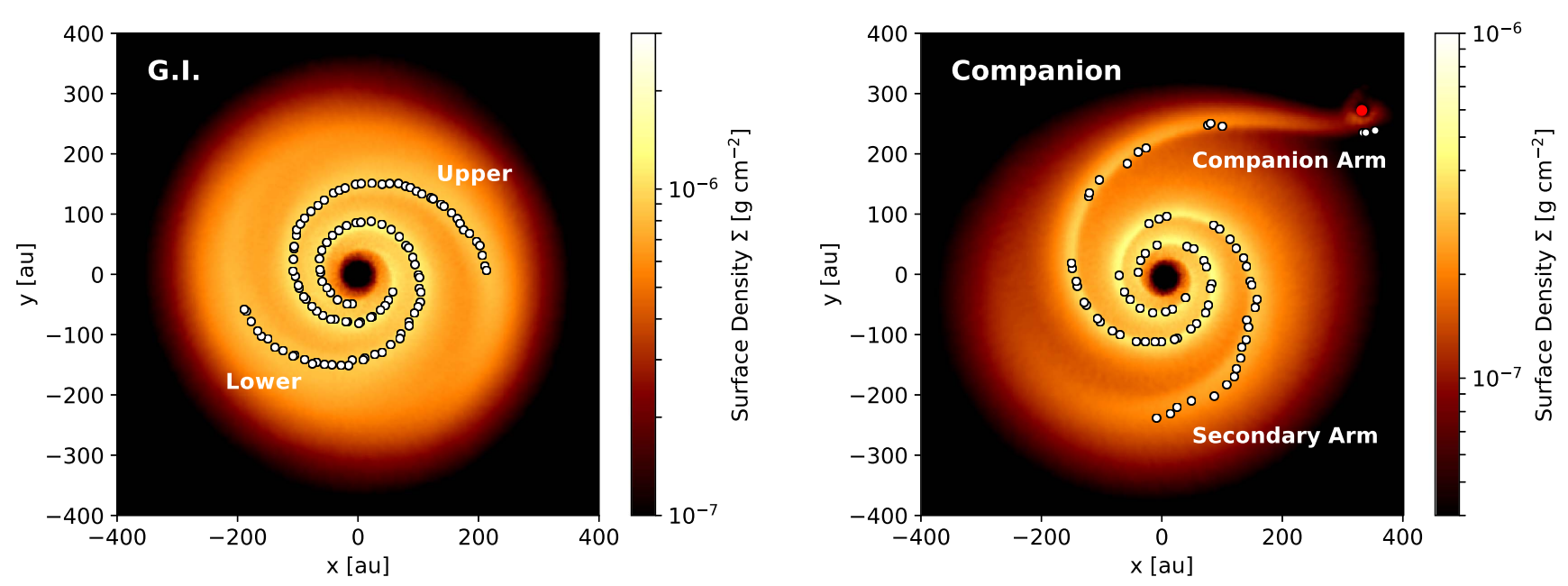

Figure 1. Surface density of the gravitationally unstable disk (left) and the companion encounter disk (right) overlaid with the results of the TACHE spiral identification algorithm (black crosses). Individual arms are identified as either "upper" or "lower" in each case.

found with the following function for $b$ (Zhu et al. 2015),

$$
b(r)=h_{p}\left(\frac{r_{p}}{r}\right)^{1+\eta} \frac{r^{\alpha}}{r^{\alpha}-r_{p}^{\alpha}},
$$

where $r_{p}$ is the orbital radius of the companion $\Omega \propto r^{-\alpha}$, $c_{s} \propto r^{-\eta}$, and $h_{p}$ is the aspect ratio of the disk at the location of the companion.

We find that this function gives a very poor fit for both cases, as the spiral arms are deeply non-linear, due to the massive, self-gravitating nature of both disks (and the relatively high mass companion). Despite the lack of theoretical guidance in this regime, we still consider the possibility that the pitch angle does vary with radius. Instead, we fit a simpler function that describes a pitch angle that increases relatively slowly with radius (VARLOG),

$$
b(r)=b_{0}+c\left(\frac{r-a}{a}\right)^{n},
$$

where $b=b_{0}$ at $r=a$, and the PURELOG solution is recovered for $c=0$. We also check specifically whether a linear dependence of $b$ with $r$ is sufficient by running fits with $n=1$ (VARLOGN). Finally, to double check that the spirals do indeed possess a logarithmic form, we also fit a power spiral (POW) function, where

$$
r=a \theta^{n}
$$

which also includes a radius-dependent pitch angle of the form

$$
\phi=n\left(\frac{r}{a}\right)^{-1 / n}
$$

where for the special cases of $n= \pm 1$, the power spiral reduces to the Archimedean and hyperbolic spirals, respectively (modulo constants describing the spiral origin).

\section{Results and Discussion}

Figure 1 shows the results of TACHE's spiral spine identification for the GI disk (left) and the Companion disk (right). Note that for the Companion disk, the spine identification slightly misses the location of the companion. This is due to the fact that the particles immediately surrounding the companion have a different tensor class to that of the spirals, and are thus removed from the spiral spine fitting.

In the following sections we attempt to fit these spiral spines using the spiral functions described previously. A summary of our best-fitting parameters for each case, along with the respective goodness-of-fit statistics, are given in Table 1.

\subsection{Isolated GI Disk}

We find that for the isolated GI disk, pure logarithmic spirals of constant pitch angle (PURELOG) deliver a very good fit to the data. Other spiral functions yield reasonable fits to the arms (Figure 2), but all yield poorer $\chi^{2}$ values than PURELOG for the upper arm, and deliver little change on the lower arm. POW fits produce quite poor fits at the inner and outer regions, strongly indicating that the arm's pitch angles are indeed constant with radius, as is the case for a PURELOG spiral. VARLOG/VARLOGN fits require extra parameters and fail to yield better fits, again indicating that a constant pitch angle is the simplest and most effective model fit to these arms.

Such a result is in accordance with our expectation that spiral arms in GI disks are density waves, and should hence be pure logarithmic spirals (Cossins et al. 2009; Hall et al. 2016). It is also in accord with TACHE's previous applications to GI disks with disk-to-star mass ratios less than 0.5 (Forgan et al. 2018b). Note that the derived pitch angles for each arm are within 0.2 (1.4\%) of each other. A hallmark of isolated GI disks are arms with extremely similar (if not identical) pitch angles.

A naive conclusion might therefore be that Elias 2-27 is an isolated GI disk, as it too possesses symmetric arms with low pitch angle. However, we must note that companion-driven simulations yield unsharp mask images that also appear to possess symmetric, tightly wound arms (Meru et al. 2017), and that Elias 2-27's arm symmetry may be an artifact of the fitting procedure (see Section 3.3). As we will see in the following section, the "ground-truth" morphology of companion-driven spirals is markedly different.

\subsection{Companion Disk}

We can see from Table 1 that PURELOG fits for the companion-driven arms are significantly poorer compared to the VARLOG/VARLOGN fits. The minimum $\chi^{2}$ solution for PURELOG achieves a reasonable fit at intermediate radii, but 
Table 1

Best-fitting Parameters for both the GI and Companion Simulation for the Spiral Functions Described in Section 2.2

\begin{tabular}{|c|c|c|c|c|c|c|c|c|c|c|c|c|c|c|c|c|}
\hline & \multicolumn{8}{|c|}{ Gravitational Instability } & \multicolumn{8}{|c|}{ Companion } \\
\hline & \multicolumn{2}{|c|}{ PURELOG } & \multicolumn{2}{|c|}{ VARLOG } & \multicolumn{2}{|c|}{ VARLOGN } & \multicolumn{2}{|c|}{ POW } & \multicolumn{2}{|c|}{ PURELOG } & \multicolumn{2}{|c|}{ VARLOG } & \multicolumn{2}{|c|}{ VARLOGN } & \multicolumn{2}{|c|}{ POW } \\
\hline & $\mathrm{Up}$ & Low & Up & Low & $\mathrm{Up}$ & Low & $\mathrm{Up}$ & Low & Up & Low & Up & Low & Up & Low & $\mathrm{Up}$ & $\overline{\text { Low }}$ \\
\hline$a$ & 29.2 & 56.9 & 111.8 & 56.2 & 110.3 & 56.8 & 55.3 & 11.5 & 64.2 & 33.0 & 39.4 & 73.3 & 46.5 & 38.5 & 8.4 & 1.9 \\
\hline$b_{0}$ & 0.208 & 0.205 & 0.191 & 0.244 & 0.123 & 0.216 & $\cdots$ & $\cdots$ & 0.288 & 0.243 & 0.208 & 0.225 & 0.148 & 0.201 & $\ldots$ & $\cdots$ \\
\hline$\phi\left[^{\circ}\right]$ & 11.75 & 11.59 & $\ldots$ & $\ldots$ & $\ldots$ & $\ldots$ & $\cdots$ & $\cdots$ & 17.0 & 14.2 & $\ldots$ & $\ldots$ & $\ldots$ & $\ldots$ & $\ldots$ & $\ldots$ \\
\hline$c$ & $\cdots$ & $\cdots$ & -0.009 & -0.029 & 0.1 & -0.006 & $\cdots$ & $\cdots$ & $\cdots$ & $\cdots$ & 0.0003 & 0.0002 & 0.014 & 0.005 & $\cdots$ & $\cdots$ \\
\hline$n$ & $\cdots$ & $\cdots$ & -0.49 & 0.35 & 1.0 & 1.0 & 0.67 & 1.26 & $\ldots$ & $\ldots$ & 2.40 & 1.92 & 1.0 & 1.0 & 1.64 & 1.14 \\
\hline$\chi^{2}$ & 1.98 & 3.98 & 3.63 & 3.22 & 3.02 & 3.26 & 3.96 & 4.13 & 7.80 & 4.52 & 3.54 & 3.85 & 4.29 & 2.39 & 9.91 & 5.03 \\
\hline
\end{tabular}

Note. In each case, "up" and "low" refer to the upper and lower arms identified in Figure 1, respectively. 

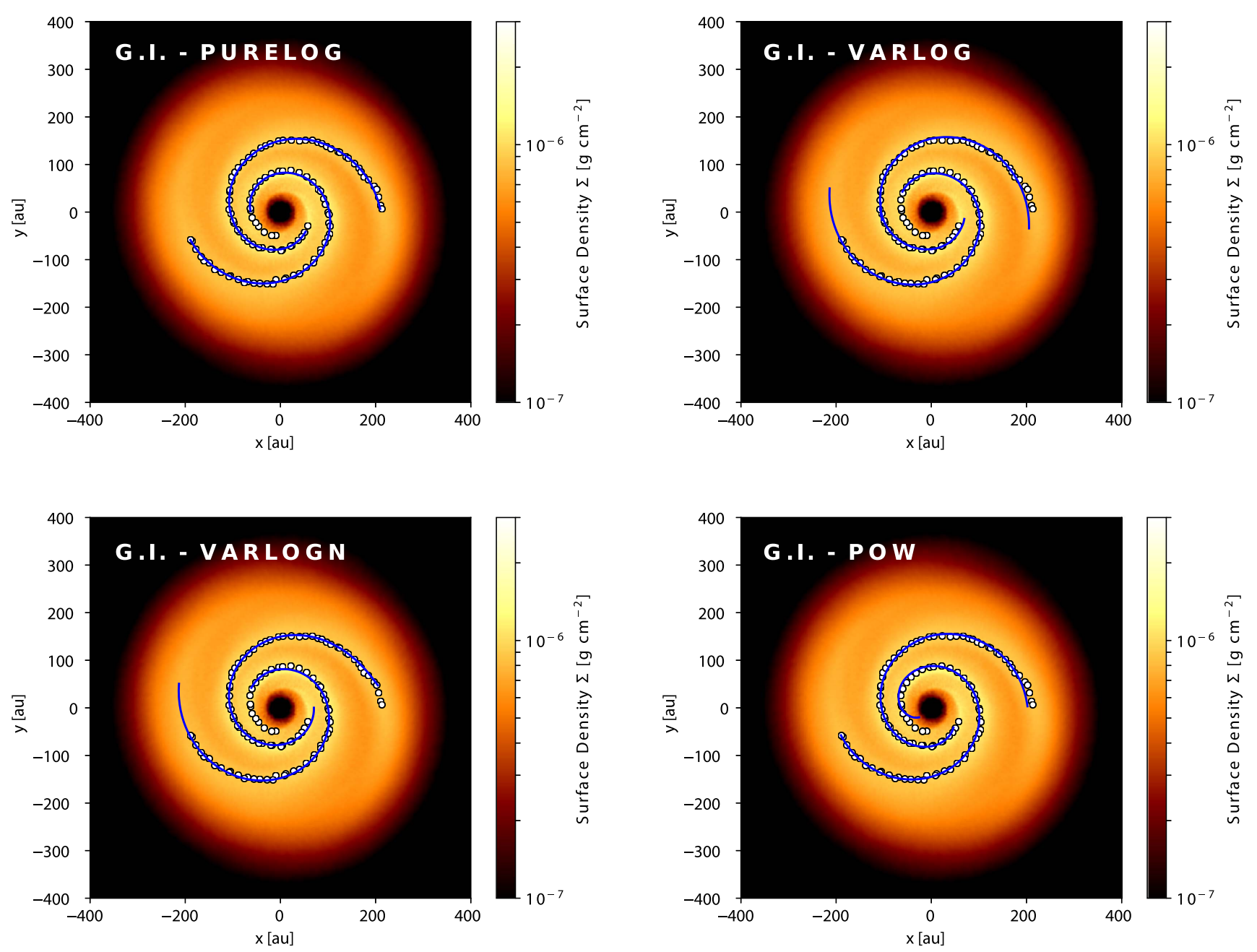

Figure 2. Isolated GI disk simulation, overlaid with the TACHE spine points and four different fitted spiral functions (see the text).

at the cost of poorly fitting points in the inner and outer disk (see Figure 3). This is heavily indicative of the fact that the pitch angle does indeed vary with radius, as is expected for companion-driven arms (Goodman \& Rafikov 2001; Rafikov 2002; Muto et al. 2012; Zhu et al. 2015).

Notably, while the companion-hosting arm is poorly fitted, the secondary arm is much better fitted, with both arms possessing larger pitch angles than the GI run. The companion arm has a pitch angle of almost $17^{\circ}$, which is (just) beyond the $10^{\circ}-15^{\circ}$ range expected for isolated GI disks (Cossins et al. 2009). The secondary arm, at $\phi \approx 15^{\circ}$, therefore appears similar to a typical GI density wave, making it less easy to distinguish.

It is worth noting that analyzing subsequent timesteps of the companion simulation yields a range of derived pitch angles for PURELOG, in some cases as large as $\sim 30^{\circ}$ for the companion arm. We find throughout that the difference in pitch angles between the companion and secondary arm are much larger than determined for pairs of arms in the GI disk (Section 3.1).

We verify that the spiral form is indeed logarithmic by considering the POW fits. We find that we cannot improve our fits (indeed, they are slightly worse). Again, we find that the best-fit power spiral fails to correctly track the location of the companion.

The best fit to the companion spirals are from the VARLOG/VARLOGN fits. For both fixed $n$ and varying $n$, we find fits to the companion arm that have $\chi^{2}$ two to three times smaller than the PURELOG and POW fits. Allowing $b$ to vary constrains the inner spiral much more effectively than the PURELOG fit, and from this analysis it seems clear that the companion arms are indeed logarithmic spirals, not power spirals. However, in contrast to the GI case, the logarithmic spirals are better fitted with a varying pitch angle as opposed to a constant pitch angle.

It is notable that for the VARLOG/VARLOGN fits our functional form for $b$ results in a minimum in $\theta(r)$ :

$$
\theta(r)=\frac{1}{b(r)} \ln \left(\frac{r}{a}\right),
$$

which occurs at

$$
\frac{d \theta}{d r}=\frac{1}{b r}-\frac{\theta}{b} \frac{d b}{d r}=0 .
$$

As

$$
\frac{d b}{d r}=n c\left(\frac{r-a}{a}\right)^{n} \frac{1}{r-a}=n \frac{b-b_{0}}{r-a} .
$$

We therefore find a minimum in $\theta(r)$ at

$$
\theta=\left(r \frac{d b}{d r}\right)^{-1}=\frac{r-a}{r} \frac{1}{n\left(b-b_{0}\right)} .
$$



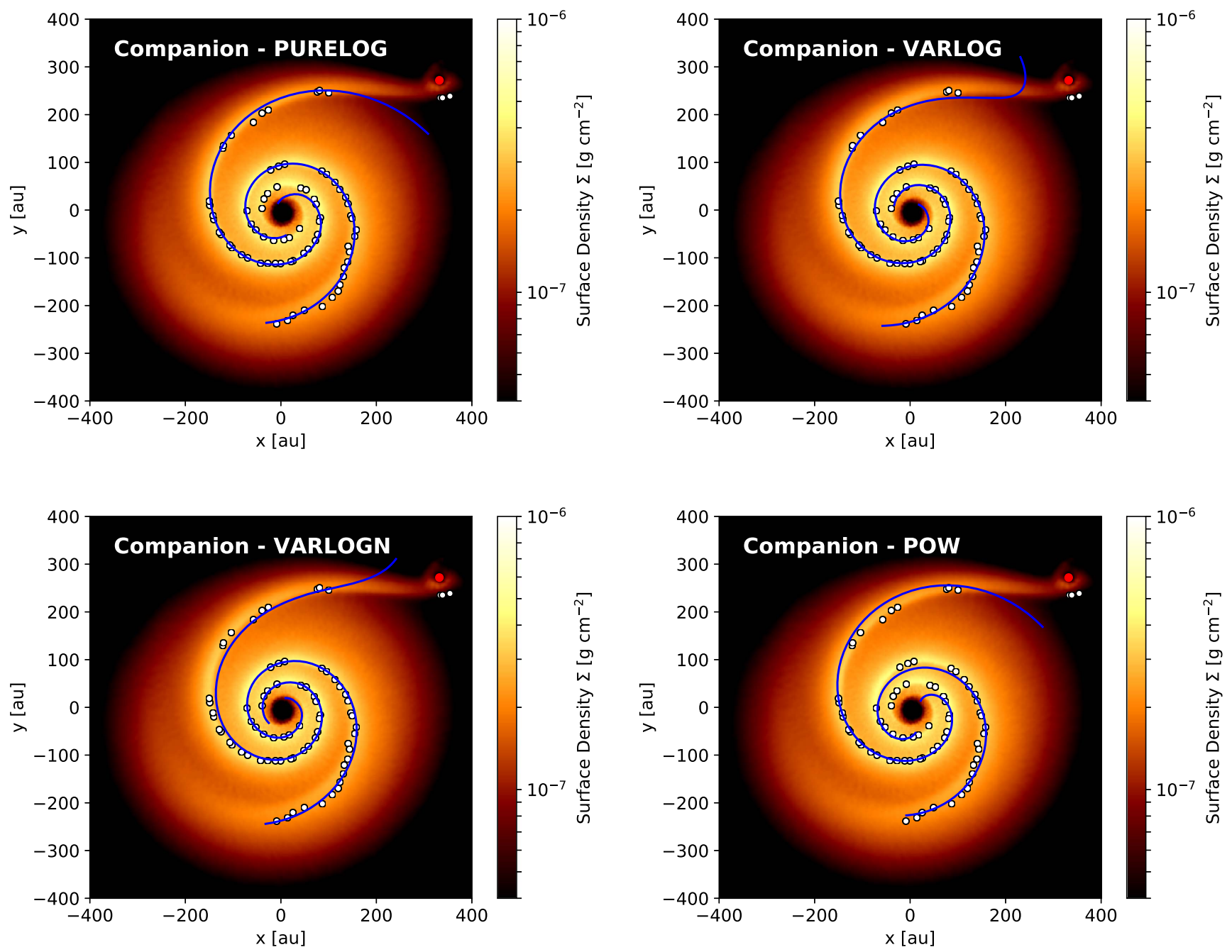

Figure 3. Same as in Figure 2, but for the Companion simulation. The location of the perturbing companion is shown in each panel by a red circle.

Hence we see that beyond this minimum, the fitted spiral function turns away from the companion. Note that attempts to fit this function on subsequent timesteps delivers a good fit to the companion arm, and not the secondary arm. Essentially, the length of the arm determines whether VARLOG/VARLOGN fits will capture the entire arm, or turn away at $\frac{d \theta}{d r}=0$.

This is an important issue for attempts to fit spiral structure driven by companions. Despite TACHE being able to roughly locate the companion inside the spiral structure, attempts to fit the structure with typical spiral functions uniformly fail to locate the companion correctly. We therefore urge caution when attempting to determine the location of an unseen companion using spiral structure alone. If the companion mass is much less than $\sim 0.02$ times the stellar mass, the two arms can be significantly asymmetric, and the companion location can be determined using the two spiral arms alone (Fung \& Dong 2015).

\subsection{Implications for Elias 2-27}

Both the GI and Companion simulations, when observed synthetically and subjected to the same unsharp mask imaging techniques as carried out by Pérez et al. (2016), broadly reproduce the observed spiral morphology of Elias 2-27 (Meru et al. 2017). However, our results identify key discriminators between the "ground-truth" spiral morphologies of the two cases.

If Elias 2-27 is an isolated, gravitationally unstable protostellar disk, then our simulations predict symmetric, logarithmic spiral arms of constant pitch $\phi \sim 12^{\circ}$; these are consistent with expectations from density wave theory (Lin \& Shu 1964; Bertin $\&$ Lin 1996), which are appropriate as for this case the disk-tostar mass ratio $q \lesssim 0.5$ (Cossins et al. 2009; Forgan et al. 2011; Hall et al. 2016). This is slightly larger than the measured pitch of $\sim 8^{\circ}$ from Pérez et al. (2016), but we should be encouraged by the fact that our simulations produce a similar pitch angle without a great deal of tuning (as the simulations by Meru et al. were not intended to reproduce the exact ALMA image, but were testing which scenarios produced morphologies that were consistent with the observations).

If Elias 2-27 is stable against GI, but undergoing encounters with an external companion, our simulations show it should produce asymmetric arms with pitch angles that vary with radius, and a larger mean pitch overall. Due to the relatively large disk mass in both simulations (and the relatively massive companion in the Companion simulation), we find that the typical expressions for companion-driven arms (e.g., Zhu et al. 2015) are a poor fit for the spirals. We also find that other spiral functions (such as the power spiral) are generally a worse fit to the data. 
The current ALMA observations indicate that Elias 2-27 does possess tightly wound, symmetric logarithmic spiral structure, suggesting that it is in fact a GI disk. However, we note that Pérez et al. (2016)'s fits to the arms assume symmetry, precluding the study of an important observable for determining the origin of spiral structure. We recommend that future observations of Elias 2-27 (and other disks with midplanedriven spiral arms) conduct fitting of individual arms rather than a single, simultaneous fit to all arms.

\section{Conclusions}

In this Letter, we have conducted a comparative spiral morphology study on massive protostellar disk simulations tuned to reproduce the observed spiral structure in the protostellar disk Elias 2-27 (Pérez et al. 2016). One simulation presented an isolated disk with $Q \lesssim 1$ resulting in GI, the other presented a disk with $Q>2$, undergoing perturbations during an encounter with a 10-Jupiter-mass companion (Companion).

Using the TACHE algorithm (Forgan et al. 2018b) on the simulation data, we identify the spine points associated with individual spiral arms for both simulations, and fit these spine points to a variety of spiral functions. Our results show that the two simulations have markedly different "ground-truth" spiral morphology, and yet produce similar "observed" morphologies under unsharp masking (Meru et al. 2017).

From this study, and from previous work, we identify several key discriminators between GI and companion-driven spiral structure for the case of Elias 2-27. The GI disk exhibits pure, symmetric logarithmic spirals of constant pitch angle, whereas the Companion disk shows asymmetric logarithmic spirals, with a pitch angle that varies with radius.

In particular, we show that asymmetry between spiral arms is a key observable, and as such observers must attempt to fit spirals individually, rather than assuming a single set of fit parameters for all spirals. It is worth noting that arm asymmetry is sensitive to the orbital phase of the companion, and this should be factored into any predictions based purely on asymmetry.

In conjunction with Meru et al. (2017), this study demonstrates that the current ALMA observations do not yet differentiate between the spiral structures observed in the GI and Companion simulations for Elias 2-27. This highlights the need for synthetic observations as a tool to both evaluate numerical simulations and to interpret real observations (c.f. Haworth et al. 2017).

In summary: we recommend further high-resolution observations of Elias 2-27 to determine the source of its spiral structure. Between the two scenarios tested here, our simulations predict morphological differences on scales of $10-20 \mathrm{au}$ in the outer regions of the disk. Therefore, future observations of Elias 2-27 with angular resolutions of tens of milli-arcseconds have the potential to determine the source of its spiral structure. We predict that if these observations continue to show tightly wound, symmetric spiral structure, then Elias 2-27 is indeed the first observed self-gravitating protostellar disk.

D.H.F. gratefully acknowledges support from the ECOGAL project, grant agreement 291227, funded by the European Research Council under ERC-2011-ADG. J.D.I. and F.M. acknowledge support from the DISCSIM project, grant agreement 341137 under ERC-2013-ADG. F.M. also acknowledges support from The Leverhulme Trust, the Isaac Newton Trust, and the Royal Society Dorothy Hodgkin Fellowship. The authors warmly thank the anonymous referee for comments that helped to clarify the manuscript. This work used the Darwin DiRAC HPC cluster at the University of Cambridge, and the Cambridge COSMOS SMP system funded by ST/J005673/1, ST/H008586/1 and ST/K00333X/1 grants. This research made use of NASA's Astrophysics Data System Bibliographic Services. This work utilised the Darwin DiRAC HPC cluster at the University of Cambridge.

Software: TACHE: https://github.com/dh4gan/tache SCIPY: http://www.scipy.org.

\section{ORCID iDs}

Duncan H. Forgan (1D https://orcid.org/0000-0003-1175-4388

John D. Ilee (1D https://orcid.org/0000-0003-1008-1142

Farzana Meru (ib https://orcid.org/0000-0002-3984-9496

\section{References}

Andrews, S. M., Wilner, D. J., Hughes, A. M., Qi, C., \& Dullemond, C. P. 2009, ApJ, 700, 1502

Benisty, M., Juhasz, A., Boccaletti, A., et al. 2015, A\&A, 578, L6

Bertin, G., \& Lin, C. C. 1996, Spiral Structure in Galaxies a Density Wave Theory (Cambridge, MA: MIT Press)

Cossins, P., Lodato, G., \& Clarke, C. J. 2009, MNRAS, 393, 1157

Crida, A., Morbidelli, A., \& Masset, F. 2006, Icar, 181, 587

Dong, R., Zhu, Z., Fung, J., et al. 2016, ApJL, 816, L12

Evans, M. G., Ilee, J. D., Hartquist, T. W., et al. 2017, MNRAS, 470, 1828

Forgan, D., Bonnell, I., Lucas, W., \& Rice, K. 2016a, MNRAS, 457, 2501

Forgan, D., \& Rice, K. 2013a, MNRAS, 432, 3168

Forgan, D., \& Rice, K. 2013b, MNRAS, 433, 1796

Forgan, D., Rice, K., Cossins, P., \& Lodato, G. 2011, MNRAS, 410, 994

Forgan, D. H., Hall, C., Meru, F., \& Rice, W. K. M. 2018a, MNRAS, 474, 5036

Forgan, D. H., Ilee, J. D., Cyganowski, C. J., Brogan, C. L., \& Hunter, T. R. 2016b, MNRAS, 463, 957

Forgan, D. H., Ramón-Fox, F. G., \& Bonnell, I. A. 2018b, arXiv:1802.01364

Fukagawa, M., Tamura, M., Itoh, Y., et al. 2006, ApJL, 636, L153

Fung, J., \& Dong, R. 2015, ApJL, 815, L21

Galvagni, M., \& Mayer, L. 2014, MNRAS, 437, 2909

Gammie, C. F. 2001, ApJ, 553, 174

Goodman, J., \& Rafikov, R. R. 2001, ApJ, 552, 793

Greaves, J. S., \& Rice, W. K. M. 2010, MNRAS, 407, 1981

Hall, C., Forgan, D., Rice, K., et al. 2016, MNRAS, 458, 306

Hall, C., Rice, K., Dipierro, G., et al. 2018, MNRAS, 477, 1004

Haworth, T. J., Glover, S. C. O., Koepferl, C. M., Bisbas, T. G., \& Dale, J. E. 2017, arXiv: 1711.05275

Isella, A., Carpenter, J. M., \& Sargent, A. I. 2009, ApJ, 701, 260

Laughlin, G., \& Bodenheimer, P. 1994, ApJ, 436, 335

Lin, C. C., \& Shu, F. H. 1964, ApJ, 140, 646

Lin, D. N. C., \& Papaloizou, J. 1986, ApJ, 309, 846

Lodato, G., \& Rice, W. K. M. 2005, MNRAS, 358, 1489

Luhman, K. L., \& Rieke, G. H. 1999, ApJ, 525, 440

Meru, F. 2015, MNRAS, 454, 2529

Meru, F., Juhász, A., Ilee, J. D., et al. 2017, ApJL, 839, L24

Muto, T., Grady, C. A., Hashimoto, J., et al. 2012, ApJL, 748, L22

Natta, A., Testi, L., \& Randich, S. 2006, A\&A, 452, 245

Pérez, L. M., Carpenter, J. M., Andrews, S. M., et al. 2016, Sci, 353, 1519

Rafikov, R. R. 2002, ApJ, 569, 997

Ricci, L., Testi, L., Natta, A., \& Brooks, K. J. 2010, A\&A, 521, A66

Rice, W. K. M., Lodato, G., \& Armitage, P. J. 2005, MNRAS, 364, L56

Stamatellos, D., Hubber, D. A., \& Whitworth, A. P. 2007, MNRAS, 382, L30

Tomida, K., Machida, M. N., Hosokawa, T., Sakurai, Y., \& Lin, C. H. 2017, ApJL, 835, L11

Toomre, A. 1964, ApJ, 139, 1217

Whitehouse, S. C., \& Bate, M. R. 2006, MNRAS, 367, 32

Whitehouse, S. C., Bate, M. R., \& Monaghan, J. J. 2005, MNRAS, 364, 1367

Zhu, Z., Dong, R., Stone, J. M., \& Rafikov, R. R. 2015, ApJ, 813, 88 